\title{
Unsteady couette flow in an annulus with combined mode of magnetic field application: A generalization
}

\author{
Michael O. Oni*, Taiwo S. Yusuf \\ Department of Mathematics, Ahmadu Bello University, Zaria, Nigeria \\ Email: michaeloni29@yahoo.com
}

\begin{abstract}
This study generalizes the role of transversely and radially applied magnetic field on flow formation in an annulus. The flow is assumed to be fully developed and driven by the movement of the cylinders. The governing momentum equation is derived and solved using the Laplace transform technique. The impact of moving inner, outer and both cylinders on flow formation is also considered. Result indicate that the application of both magnetic field leads to a further decrease in fluid velocity and an increase in skin-friction at the inner surface of outer cylinder. In addition, the movement of the cylinders is significant in the attainment of steady state skin-friction at the moving wall.
\end{abstract}

Keywords: Transverse Magnetic Field, Radial Magnetic Field, Annulus, Unsteady, Couette Flow.

\section{INTRODUCTION}

The significant of magneto hydrodynamics (MHD) in nature and industrial applications cannot be over emphasized. This dynamic insights has made scientist to venture more into understanding role of MHD in solving physical situations that arises daily Li et al. [1]. Some of these applications include the magnetic behavior of plasmas in fusion reactors, liquid-metal cooling of nuclear reactors and electromagnetic casting. Many research work has been committed to study the effect of transversely externally applied magnetic field on fluid flow in annulus or micro-annulus Rossov [2], Hartmann [3], Jha and Odengle [4], Jha and Aina [6]. They found interesting result that the role of magnetic field is to decrease fluid motion as well as skin-friction. In other works (Nandi [6], Jha and Oni [7] and Makinde et al. [8]), the magnetic field are assume to be radially applied to flow direction. Similar results on flow formation as well as skin-friction are also obtained.

On the other hand, the study of Couette flow in annular geometry has an increasing daily application in medicine, disease control and automobiles; movement of piston. A lot of work has been carried out to have a deeper understanding of the role of movement of one of the cylinder (Katagiri [9], Jha and Apere [10], Farhad [11]).

In all the studied literature, no work has been devoted to see the effect of combined (transverse and radial) magnetic field. Also, it not very clear which cylinder should be moving in order to increase or decrease the force at which the fluid hits the surfaces of the cylinders (skin-friction).

Therefore, this current research aimed at investigating the combined effect of transverse and radial mode of magnetic field application on Couette flow formation and skin-friction in an annulus. The governing momentum equation governing the flow formation for the case of transverse and radial magnetic field is generalized as a single equation.

\section{MATHEMATICAL ANALYSIS}

Consider the motion of a viscous, laminar, incompressible and electrically conducting fluid filling the gap between two concentric cylinders. The fluid exists in the region $a \leq r \leq b$, where the $r$ axis is the coordinate normal to the flow and $a, b$ are the radiuses of the inner and outer cylinders respectively. The fluid flow inside the annulus is induced by movement of the inner or outer cylinder which is located at $r=a, r=$ $b$ respectively. Since the cylinders are of infinite length and the flow is fully developed, all physical parameters are functions of $r$ and $t^{\prime}$. It is further assumed that no applied and polarisation voltage exists. The flow field is exposed to the influence of an externally applied transverse or (and) radial magnetic field. It is assumed that the magnetic Reynolds number is very small, which corresponds to negligibly induced magnetic field compared to the externally applied one (Pai [12]; Jha and Apere [13]. Under these assumptions, the unsteady hydrodynamic continuity and momentum equations in the component form in the absence of pressure gradient are respectively:

$\frac{d u}{d r}=0$ 
$\frac{\partial u}{\partial t^{\prime}}=\frac{v}{r}\left[\frac{\partial}{\partial r}\left(r \frac{\partial u}{\partial r}\right)\right]-\frac{\sigma B_{0}^{2}}{\rho} u$

$\frac{\partial u}{\partial t^{\prime}}=\frac{v}{r}\left[\frac{\partial}{\partial r}\left(r \frac{\partial u}{\partial r}\right)\right]-\frac{\sigma B_{0}^{2}}{\rho r^{2}} u$

subject to

$t^{\prime} \leq 0 u=0 a \leq r \leq b$

$t^{\prime}>0$

$\left\{\begin{array}{l}u=A u_{0} \\ u=B u_{0}\end{array}\right.$

$r=a$

$r=b$

Using the following dimensionless parameters, the governing equation is obtained as:

$R=\frac{r}{a}, \lambda=\frac{b}{a}, \quad u=\frac{U}{u_{0}}, M^{2}=\frac{\sigma \mu_{e}^{2} B_{0}^{2} a^{2}}{\mu}, t=\frac{t^{\prime} v}{a^{2}}$

$\frac{\partial U}{\partial t}=\frac{1}{R}\left[\frac{\partial}{\partial R}\left(R \frac{\partial U}{\partial R}\right)\right]-M^{2} U$

$\frac{\partial U}{\partial t}=\frac{1}{R}\left[\frac{\partial}{\partial R}\left(R \frac{\partial U}{\partial R}\right)\right]-\frac{M^{2} U}{R^{2}}$

$t \leq 0 U=01 \leq R \leq \lambda$

$\begin{array}{lll}t>0 & \begin{cases}U=A & R=1 \\ u=B & R=\lambda\end{cases} \end{array}$

Unifying equations (6) and (7),

$\frac{\partial U}{\partial t}=\frac{1}{R}\left[\frac{\partial}{\partial R}\left(R \frac{\partial U}{\partial R}\right)\right]-M^{2} U\left[C+\frac{D}{R^{2}}\right]$

where $A, B, C$ and $D$ are constants that assume value zero or one as follow:

$C=0, D=0 ;$ no applied magnetic field

$C=1, D=0$; transversely applied magnetic field only

$C=0, D=1$; radially applied magnetic field only

$C=1, D=1$; combined transversely and radially applied magnetic field

The solution of equation (9) can be obtained using the Laplace transform technique. Define the following transform variables

$\bar{U}(R, S)=\int_{0}^{\infty} U(R, t) e^{-S t} d t$, where $S$ is the Laplace parameter and $S>0$

And taking the Laplace transform of Eq. (9), we obtain the following ordinary differential equation

$\frac{1}{R}\left[\frac{\partial}{\partial R}\left(R \frac{\partial \bar{U}}{\partial R}\right)\right]-M^{2} \bar{U}\left[(C+S)+\frac{D}{R^{2}}\right]$

Solving equation (10) with boundary conditions (8), the closed form expression in Laplace domain is obtained:

$\bar{U}(R, S)=$

$\frac{\left[A K_{D_{1}}\left(\lambda D_{2}\right)-B K_{D_{1}}\left(D_{2}\right)\right] I_{D_{1}}\left(D_{2} R\right)+\left[B I_{D_{1}}\left(D_{2}\right)-A I_{D_{1}}\left(\lambda D_{2}\right)\right] K_{D_{1}}\left(D_{2} R\right)}{S\left[I_{D_{1}}\left(D_{2}\right) K_{D_{1}}\left(\lambda D_{2}\right)-I_{D_{1}}\left(\lambda D_{2}\right) K_{D_{1}}\left(D_{2}\right)\right]}$ where $C_{1}, C_{2}, D_{1}$ and $D_{2}$ are constants and $S$ is the Laplace domain:

$$
\begin{array}{ll}
D_{1}=M \sqrt{D} \quad, \quad D_{2}=\sqrt{M^{2} C+S} \quad, \quad C_{1}= \\
\frac{\left[A K_{D_{1}}\left(\lambda D_{2}\right)-B K_{D_{1}}\left(D_{2}\right)\right]}{S\left[I_{D_{1}}\left(D_{2}\right) K_{D_{1}}\left(\lambda D_{2}\right)-I_{D_{1}}\left(\lambda D_{2}\right) K_{D_{1}}\left(D_{2}\right)\right]} \text { and } & C_{2}= \\
\frac{\left[B I_{D_{1}}\left(D_{2}\right)-A I_{D_{1}}\left(\lambda D_{2}\right)\right]}{S\left[I_{D_{1}}\left(D_{2}\right) K_{D_{1}}\left(\lambda D_{2}\right)-I_{D_{1}}\left(\lambda D_{2}\right) K_{D_{1}}\left(D_{2}\right)\right]} & \text { (12) }
\end{array}
$$

The skin-friction at the surfaces of the cylinders is obtained as follows:

$\left.\frac{d \bar{U}(R, S)}{d R}\right|_{R=1}=$
$D_{2}\left[\frac{\left[A K_{D_{1}}\left(\lambda D_{2}\right)-B K_{D_{1}}\left(D_{2}\right)\right] I_{\left(D_{1}-1\right)}\left(D_{2}\right)-\left[B I_{D_{1}}\left(D_{2}\right)-A I_{D_{1}}\left(\lambda D_{2}\right)\right] K_{\left(D_{1}-1\right)}\left(D_{2}\right)}{S\left[I_{D_{1}}\left(D_{2}\right) K_{D_{1}}\left(\lambda D_{2}\right)-I_{D_{1}}\left(\lambda D_{2}\right) K_{D_{1}}\left(D_{2}\right)\right]}\right]$

$\left.\frac{d \bar{U}(R, S)}{d R}\right|_{R=\lambda}=$

$D_{2}\left[\frac{\left[A K_{D_{1}}\left(\lambda D_{2}\right)-B K_{D_{1}}\left(D_{2}\right)\right] I_{\left(D_{1}-1\right)}\left(\lambda D_{2}\right)-\left[B I_{D_{1}}\left(D_{2}\right)-A I_{D_{1}}\left(\lambda D_{2}\right)\right] K_{\left(D_{1}-1\right)}\left(\lambda D_{2}\right)}{S\left[I_{D_{1}}\left(D_{2}\right) K_{D_{1}}\left(\lambda D_{2}\right)-I_{D_{1}}\left(\lambda D_{2}\right) K_{D_{1}}\left(D_{2}\right)\right]}\right]$

It is good to note that the solutions above are in Laplace domain. It is therefore significant to transform to time domain. Due to the complexity of these solution, the Riemann-sum approximation which a promising too for accuracy [13] is used to transform equations (10-13) the Laplace domain into time domain as follows:

$U(R, t)=\frac{e^{\varepsilon t}}{t}\left[\frac{1}{2} \bar{U}(R, \varepsilon)+\operatorname{Re} \sum_{n=1}^{N} \bar{U}(R, \varepsilon+\right.$

$\left.\left.\frac{i n \pi}{t}\right)(-1)^{n}\right], 1 \leq R \leq \lambda$

where $R e$ refers to the real part of $i=\sqrt{-1}$ the imaginary number. $N$ is the number of terms used in the Riemann-sum approximation and $\varepsilon$ is the real part of the Bromwich contour that is used in inverting Laplace transforms. The Riemann-sum approximation for the Laplace inversion involves a single summation for the numerical process its accuracy depends on the value of $\varepsilon$ and the truncation error dictated by $N$. According to Tzou [14], the value of $\varepsilon t$ that best satisfied the result is approximately 4.7 .

\section{RESULTS AND DISCUSSIONS}

This section is devoted to the graphical representation of solutions obtained in previous section. The flow formation is seen to be regulated by Laplace parameter $(S)$, Hartmann number $(M)$, annular gap $(\lambda)$ and movement of the inner and outer cylinders respectively $(A=1, B=0$ or $A=0, B=1)$. In addition, $C$ and $D$ are constants which assume 1 and 0 indicating the presence or absence of magnetic field (M.F) respectively. 


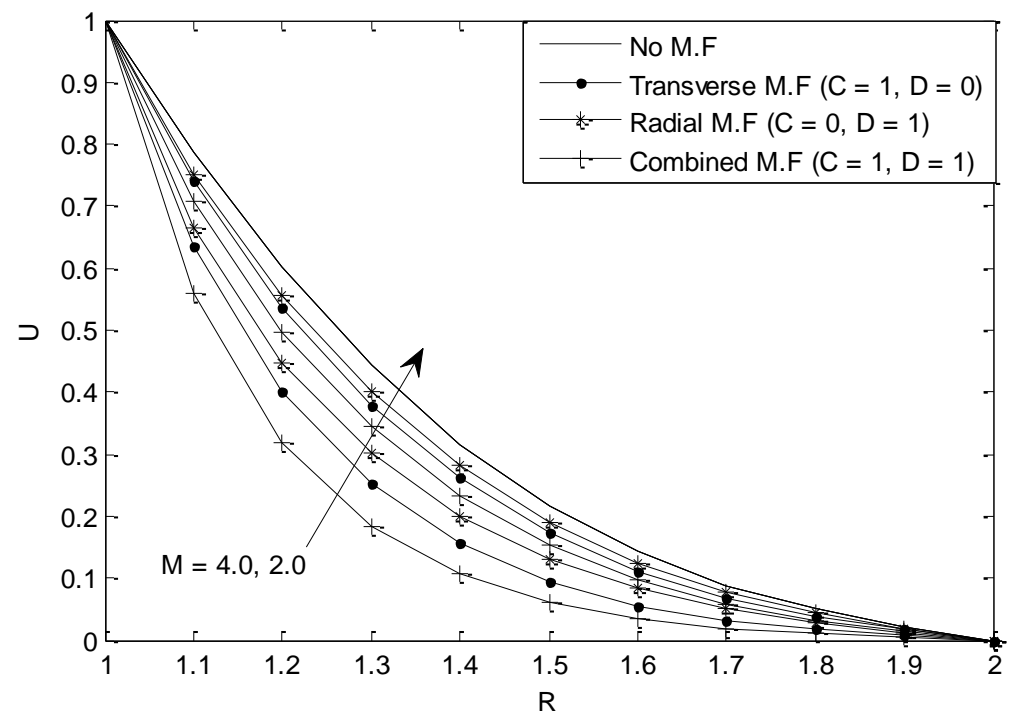

Figure 1a: Velocity profile for different values of $\mathrm{M}$ and M.F application at $t=0.1$ $\lambda=2.0, A=1, B=0$

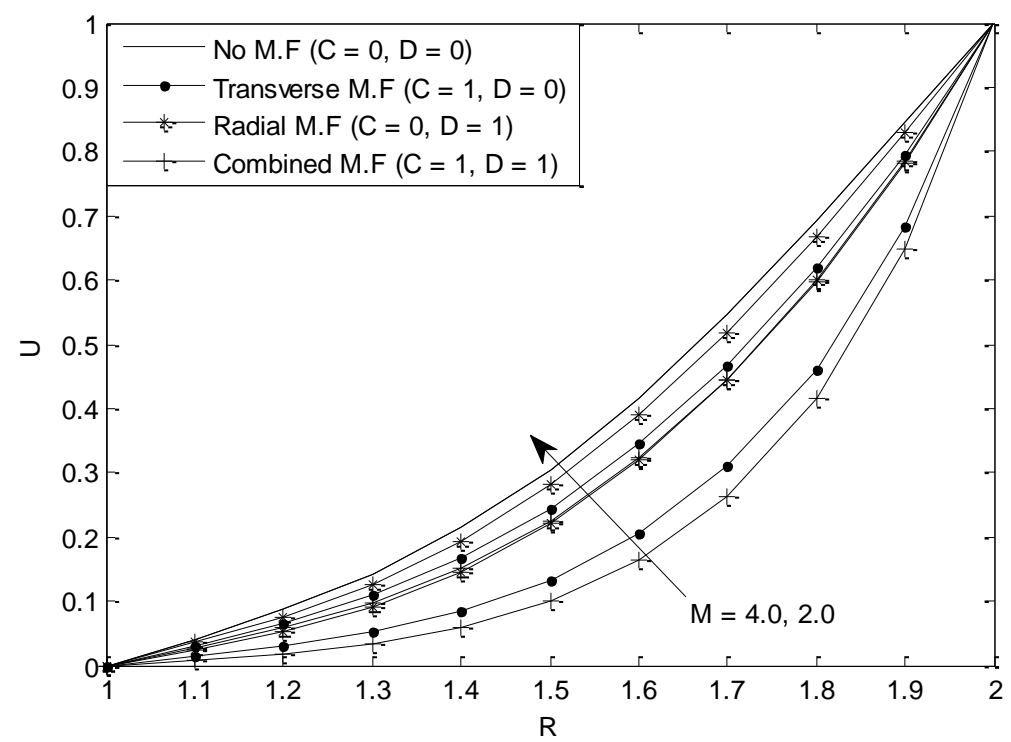

Figure 1b: Velocity profile for different values of $\mathrm{M}$ and M.F application at $\mathrm{t}=0.1$ $\lambda=2.0, A=0, B=1$

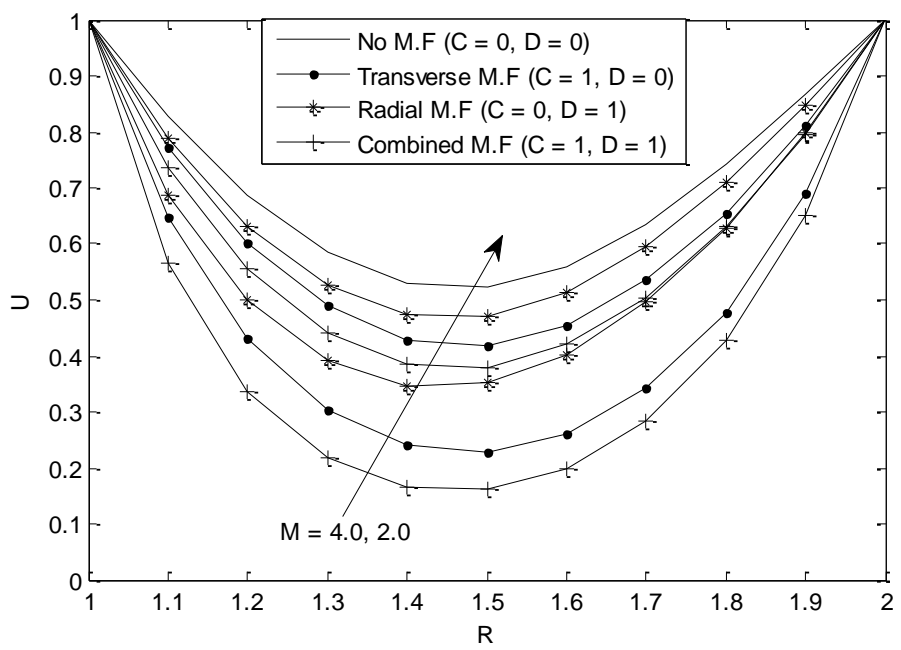

Figure 1c: Velocity profile for different values of $\mathrm{M}$ and M.F application at $\mathrm{t}=0.1$, $\lambda=2.0, A=1, B=1$ 
Figures 1a-1c depict the role of transversely, radially or combined magnetic field on fluid velocity at fixed value $\lambda$ and $t$ for the case when the inner, outer and both cylinders are moving respectively. It is found that the maximum velocity is obtained when both cylinders are moving (Fig. 1c). As expected, the role of $M$ is observe to decrease fluid velocity. Thus, the application of combined magnetic field further retards fluid motion for all cases of movement of cylinders (Fig. 1a-1c). Further, there is no significant difference whether the inner or outer cylinder is the moving cylinder. In addition, radially applied magnetic field has a higher velocity compared to the case of transversely applied magnetic field. This is significant as it can be used as a control mechanism for fluid velocity in industries when there is need to increase or decrease motion of the fluid.

Figures $2 a-2 c$ on the other hand present the effect of magnetic field and movement of the cylinder on skin-friction at the inner surface of outer cylinder at different time in the annulus for the case when the inner, outer and both cylinders are moving respectively. Result shows that the role of combined magnetic field leads to an increase in the skinfriction at this wall. Also, it is found that skin-friction increases with increase in $M$. This is due to the presence of Lorentz force in the magnetic field. In addition, the movement of inner cylinder leads to faster attainment of steady state skin-friction compared to the case of movement of both cylinders.

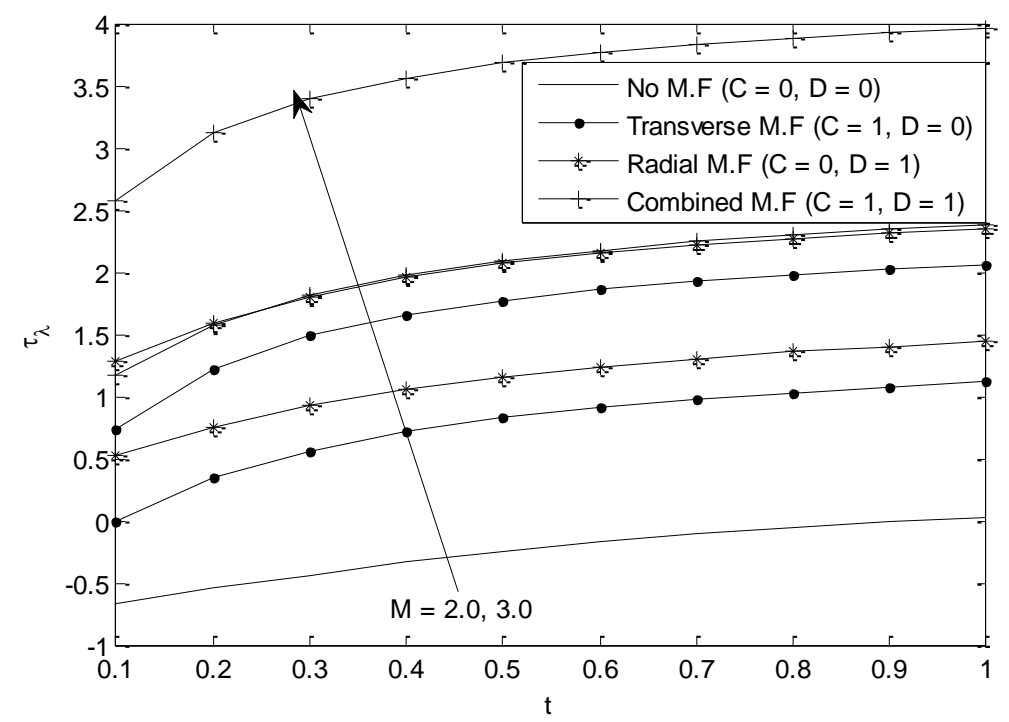

Figure $2 b$ : Skin-friction for different values of $M$ and M.F application at $\lambda=2.0, A=0, B=1(R=\lambda)$

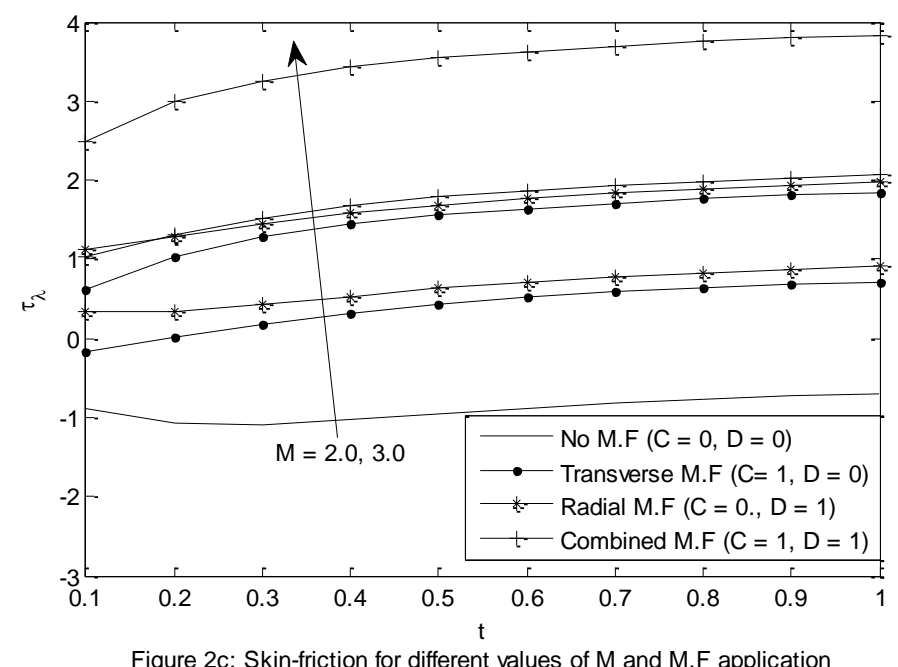

Figure 2c: Skin-friction for different values of $M$ and M.F application at $\lambda=2.0, A=1, B=1(R=\lambda)$

\section{CONCLUSION}

This study generalized the role of transversely and radially magnetic field application on flow formation in an annulus. The flow is assumed to be fully developed and driven by the movement of the cylinders. The governing equation is derived and solved using the Laplace transform technique. Results show that the role of combined effect of magnetic field is significant in increasing or decreasing fluid velocity or skinfriction and also in attainment of steady state. In addition, the movement of the inner, outer or both cylinders contributes adversely to the attainment of steady state skin-friction at the moving surface. The findings in this article can serve as a control mechanism in situations when there is need to increase 
or decrease fluid velocity as well as skin-friction. The result presented in this article corresponds with the findings of Jha and Apere [13] by relaxing certain parameters $(A=1, B=$ $0, C=1, D=0$ )

\section{REFERENCES}

[1] Li Y., Wang D.K., Li Q. (2013). Application of Magneto Hydrodynamics (MHD) in hypersonic vehicle, Advanced Material Research, Vol. 631-632, pp. 890-893.

[2] Rossov V.J. (1957). Flow of electrically conducting fluids over a flat plate in the presence of a transverse magnetic field, Report 1358 National Advesory Committee for Aeronautics.

[3] Hartmann J. (1937). Hg-Dyanmics I- Theory of laminar flow of an electrically conductive liquid in a homogeneous magnetic field, $\mathrm{Kgl}$. Danske Videnskabernes Salskab, Mathematisk-Fysiske Meddelelser, Vol. 15, No. 6, Copenhagen.

[4] Jha B.K., Odengle J.O. (2015). Unsteady MHD Couette flow in composite channel partially filled with porous material: A semi-analytical approach, Transp Porous Med, Vol. 107, pp. 219 - 234.

[5] Jha B.K., Aina B. (2015). Magnetohydrodynamics of a mixed convection flow in a vertical microannulus: An exact solution, Int. J. Of Fluid Mechanics Research, Vol. 42, No. 6, pp. 537-552. DOI: 10.1615/interJFluidMechRes.V42.16.40

[6] Nandi S. (1973). MHD flow in an annulus with porous walls under an external radial magnetic field, Pageoph, Vol. 105, p. 825.

[7] Jha B.K., Oni M.O. (2017). Impact of mode of application of magnetic field on rate of heat transfer of rarefied gas flow in a microtube, Alexandria Engr. J., DOI: 10.1016/j.aej.2017.03.029

[8] Makinde O.D., Bég O.A., Takhar H.S. (2009). Magnetohydrodynamic viscous flow in a porous medium cylindrical annulus with an applied radial magnetic field, Int J Appl Math Mech, Vol. 5, pp. 6881.

[9] Katagiri M. (1962). Flow formation in Couette motion in Magnetohydrodynamics, J. Phys. Soc. Jpn. Vol. 17, pp. 393-396.

[10] Jha B.K., Apere C.A. (2013). Time-dependent MHD Couette flow in a porous annulus, Commun. Nonlinear Sci. Numer. Simul, Vol. 18, pp. 1959-1969.
[11] Farhad A., Norzieha M., Sharidan S., Khan I., Samiuthaq Q. (2012). On hydromagnetic rotating flow in a porous medium with slip condition and hall current, Int. J. Phys. Sci., Vol. 7, Vol. 10, pp. 1540-1548.

[12] Pai S.I. (1962). Magnetogasdynamics and plasma dynamics, Springer, Berlin.

[13] Jha B.K., Apere C.A. (2010). Unsteady MHD Couette flow in Annuli: the Riemann-sum approximation, Approach. J. Phy. Soc. Jpn, Vol. 79, No. 12, pp. 124403-124403-5.

[14] Tzou D.Y. (1997). Macro to microscale heat transfer: the lagging behaviour, Taylor and Francis, Washington.

\section{NOMENCLATURE}

aradius of the inner cylinder

bradius of the outer cylinder

RSARiemann-sum approximation

$I_{n} \quad$ modified Bessel function of the first kind of order $n$

$K_{n} \quad$ modified Bessel function of the second kind of order $n$ $r$ dimensional radial coordinate

$R$ dimensionless radial coordinate

$t^{\prime}$ dimensional time

$t$ dimensionless time

$u$ dimensional axial velocity

$U$ dimensionless axial velocity

\section{Greek letters}

$\alpha$ thermal diffusivity

$\lambda$ ratio of radiuses $(b / a)$

$v$ fluid kinematic viscosity

$\mu$ dynamic viscosity

$\rho$ density

$\tau$ skin-friction

$\sigma$ electrical conductivity of the fluid

$\mu_{e}$ magnetic permeability

$M$ Hartmann number

Compliance with Ethical Standards: Funding: This study was not sponsor by anyone

Conflict of Interest: Author has declared there is no conflict of interest and has not received any funding anywhere 